\title{
Complicações no tratamento com laser endovascular em varizes de membros inferiores
}

\author{
Complications evidenced in the endovascular laser treatment for varicose veins \\ Jorge E nrique Soracco ${ }^{1}$, Jorge Lopez D 'Ámbola ${ }^{1}$, José Luis Ciucci ${ }^{1}$, \\ José M aria Pereira de G odoy², Cleusa Ema Q uilici Belczak ${ }^{3}$
}

\begin{abstract}
Resumo
O bjetivo: 0 objetivo do presente estudo é relatar as complicações no tratamento de varizes em membros inferiores com laser endovascular.

M étodos: Foram levantadas, no período de junho de 1999 a de zembro de 2002, algumas complicações, como queimadura de pele, neurite do nervo safeno, hiperpigmentação e fibrose no local da safena em 250 pacientes submetidos a tratamento endovascular com laser em varizes de membros inferiores. 0 diagnóstico das complicações foi clínico e baseado nos sinais e sintomas. Avaliou-se 196 pacientes do sexo feminino e 54 do sexo masculino, com idades variando entre 25 e 79 anos, no H ospital M ilitar de Buenos Aires. Foram tratados com laser de diodo de alta potência de $810 \mathrm{~nm}$ de longitude de onda mediante um sistema de fibras óticas semi-rígidas de quartzo de 400 e $600 \mu \mathrm{m}$ e ponta de contato plana em modo cirúrgico contínuo. Para análise estatística, foram calculadas as percentagens.

Resultados: Lesões tipo queimadura foram observadas em 3,2\%, hiperpigmentação em 9,6\%, fibrose no local da safena por mais de 6 meses em $5,6 \%$ e neurite do nervo safeno em $4,8 \%$.

C onclusão: Conclui-se que o tratamento com laser endovascular de varizes de membros inferiores não é desprovido de intercorrências e que os fatores que levaram a essas complicações devem ser identificados e reavaliados.
\end{abstract}

Palavras-chave: lasers, varizes, membros inferiores.

\begin{abstract}
O bjective: The aim of the present study is to report the complications seen in the endovascular laser treatment of varicose veins of the lower limbs.

Methods: From June 1999 to D ecember 2002, 250 patients submitted to the endovascular treatment of varicose veins of the lower limbsusing lasers and suffering from complications, such as skin burns, saphenous neuritis, hyperpigmentation and fibrosis along the course of the saphen ous vein, were assessed. The diagnosis of the complications was made clinically based on the signs and symptoms evidenced. Of the 250 patients, aged $25-79$ years, treated in the $\mathrm{H}$ ospital M ilitar de Buenos Aires, 196 were female and 54 were male. H igh power $810 \mathrm{~nm}$ wavelength laser diodes were applied using semi-rigid 400 and 600 micron fiber-optic quartz systems in continuous contact with the skin. Percentages were used for statistical analysis.

Results: Burn-type injuries were observed in 3.2\% of cases, hyperpigmentation in $9.6 \%$, fibrosis along the course of the saphenous vein over at least 6 months in $5.6 \%$ and saphenous neuritis in $4.8 \%$.

Conclusion: We concluded that the endovascular laser treatment for varicose veins of the lower limbs is not free from complications; therefore, all factors involved should be identified and assessed.
\end{abstract}

Key words: lasers, varicose veins, lower extremities.

A cirurgia convencional é aceita amplamente como terapia padrão no tratamento das varizes, embora seja associada com alta recorrêncial ${ }^{1}$. Alternativamente, há a possibilidade de usar procedimentos endovasculares, como obliteração endovenosa por radiofreqüência, o laser de endovenoso e a escleroterapia, que teriam a vantagem de ser minimamente invasivos ${ }^{1}$. 
0 laser ganha crescente aceitação nos tratamentos das telangiectasias e das veias varicosas, e sugere-se que as abordagens desses vasos, durante a mesma sessão de tratamento, possam ser complementaresem indivíduos selecionados ${ }^{2,3}$, além de ser uma forma efetiva e segura no tratamento da incompetência da veia safena $a^{4-7}$.

Contudo, as seguintes complicações são citadas: queimadura de pele, neurite do nervo safeno, hiperpigmentação e fibrose no local da veia safena interna ${ }^{5-7}$. A identificação dessas complicações é importante ferramenta para esclarecer os fatores envolvidos.

0 objetivo do presente estudo é relatar complicaçõesno tratamento endovascular com laser devarizes de membros inferiores no serviço.

\section{M aterial e método}

Foram avaliadas, no período de junho de 1999 a dezembro de 2002, as complicações queimadura de pele, neurite do nervo safeno, hiperpigmentação e fibrose no local da veia safena em 250 pacientes submetidos a tratamento endovascular com laser em varizes de membros inferiores, com seguimento mínimo de 1 mês e máximo de 3 anos no Serviço $\mathrm{H}$ ospital M ilitar de Buenos Aires. 0 diagnóstico das complicações foi clínico, baseado nos sinais e sintomas. Foram tratados 196 pacientes do sexo feminino e 54 do sexo masculino, com idades variando entre 25 e 79 anos. A insuficiência venosa foi diagnosticada com eco-D oppler colorido, sendo os critérios de exclusão os portadores de insuficiência venosa crônica associada a transtornos tróficos, úlceras, arteriopatias periféricas, enfermidades crônicas, trombose venosa profunda, gestação ou al eitamento.

0 procedimento foi ambulatorial, porém permaneceram no hospital por 3 a 4 horas no pós-operatório. Foram 206 procedimentos bilaterais e 44 unilaterais, sendo feitas as ligaduras da croça da safena com anestesia local e aplicação intravenosa de laser de diodos de alta potência de $810 \mathrm{~nm}$ delongitude de onda, medianteum sistema defibrasóticassemi-rígidas dequartzo de 400 e $600 \mu \mathrm{m}$, ponta de contato plana em modo cirúrgico contínuo. Procedeu-se à marcação das veias insuficientes com os pacientes em bipedestação utilizando-se lápis dermográfico. A veia safena magna foi puncionada no pré-maléolo com agulha $18 \mathrm{G}$, e introduziu-se a fibra ótica de quartzo semi-rígido précarbonizada de $600 \mu \mathrm{m}$. Q uando não foi possível realizar a punção distal, acessou-se a veia safena pré-maleolar mediante dissecção delicada, enfatizando-se que costuma ser sempre importante certificar-se de que a fibra ótica esteja dentro da veia, devendo ascender pela mesma sem nenhuma dificuldade. Verificou-se, por transdermoiluminação, a progressão do ponteiro laser (vermelho, de diodo de $635 \mathrm{~nm}$ ) até a prega inguinal, emergindo pela safena distal seccionada e reparada ou atéo ponto de refluxo previamente marcado ediagnosticado com o eco-Doppler, como, por exemplo, a perfurante do canal de $\mathrm{H}$ unter. $\mathrm{N}$ os casos necessários, o procedimento foi realizado sob controle com ecoD oppler.

Procedeu-se de igual forma com as colaterais insuficientes e perfurantes, realizando as punções que se fizeram necessárias para conseguir todo o tratamento das varizes e bloquear os pontos de refluxo, variando as potências entre 4 a $10 \mathrm{~W}$. 0 s tratamentos foram geralmente controlados por eco-D oppler.

0 desenvolvimento dessa técnica foi aprovado pelo Comitê de Bioética do Hospital M ilitar de Buenos Aires, onde todos os pacientes foram devidamente informados sobre o método e assinaram termo de consentimento.

Para análise estatística, foram calculadas as percentagens.

\section{Resultado}

Lesões tipo queimadura foram observadas em $3,2 \%$, hiperpigmentação em 9,6\%, fibrose no local da safena por mais de 6 meses $5,6 \%$ e neurite do nervo safeno em $4,8 \%$.

\section{D iscussão}

0 presente estudo mostra que a terapia endoluminal com laser para varizes superficiais de membros inferiores pode apresentar intercorrências, como a queimadura de pele, neurite do nervo safeno, fibrose no traj eto do vaso, hiperpigmentação ehematoma, as quais geralmente melhoram com o passar dos dias. São descritas intercorrências variáveis de um serviço para outro 3,5,8,9. A parestesia é relatada em torno de $8,5 \%$ na primeira semana, em geral com regressão dentro de 6 meses, persistindo em $0,7 \%$ dos casos $^{7}$. 0 utro estudo relata parestesia em $10 \%$ dos casos no sexto mês de pós-operatório e lesão de queimadura de pele em $3,3 \%{ }^{8}$. É possível que essas diferenças sejam associadas também ao grau de experiência da 
equipe 9 . 0 utros estudos mostram freqüências maiores de complicações: parestesia local em 36,5\%, equimose em $23 \%$, queimadura superficial em $4,8 \%$, flebites superficiais em $6 \%$ ehematoma em $0,8 \%$ dos casos, sendo que não foram registradas morbidade ou mortalidade significantes. T odos os pacientes tiveram rápida recuperação, e os autores acharam que foram minimizadas as complicações depois de endovenouslaser therapy (EVLT), em comparação ao póscirúrgico convencional ${ }^{10}$. 0 que se observa nos registros da literatura é uma variação da freqüência dessas complicações, provavelmente decorrente de vários fatores. A escolha do vaso a ser tratado (maior ou menor calibre), o laser a ser utilizado (diodo) e a energia a ser despendida em cada procedimento podem ser fatores que talvez influenciem na ocorrência dessas complicações. À medida que esses fatores são identificados, é possível a redução dessas intercorrências.

Por outro lado, o laser endovascular tem semostrado eficiente quanto à resolubilidade do quadro varicoso ${ }^{5-7}$, além de ser intervenção menos agressiva em relação à cirurgia convencional ${ }^{3-7}$. A maioria das intercorrências é reversível com o passar do tempo, embora gerem desconforto temporário para os pacientes.

\section{C onclusão}

Conclui-se que o tratamento com laser endovascular devarizes demembrosinferioresnão édesprovido de complicações e que os fatores que levaram a essas complicações devem ser identificados e reavaliados.

\section{R eferências}

1. Rass K. M odern aspects in varicose vein surgery. $H$ autarzt. 2005;56:448-56.

2. Viarrengo LM, M eirelles GV, Potério Filho J. Resultados do tratamento devarizescom laser endovenoso: estudo prospectivo com segmento de 39 meses. J V asc Br. 2005,4 (Supl 1):S19.

3. Sadick N S. Laser treatment of leg veins. Skin Therapy Lett. 2004;9:6-9.

4. Perkowski P, Ravi R, Gowda RC, et al. Endovenous laser ablation of the saphenous vein for treatment of venous insufficiency and varicose veins: early results from a large single-center experience. J Endovasc Ther. 2004;11:132-8.

5. Lurie $F, C$ reton D, Eklof $B$, et al. Prospective randomized study of endovenous radiofrequency obliteration (closure procedure) versus ligation and stripping in a selected patient population (EV O LV eS Study). J V asc Surg. 2003;38:207-14.

6. M erchant RF, DePalma RG, Kabnick LS. Endovascular obliteration of saphenous reflux: a multicenter study. J Vasc Surg. 2002;35:1190-6.

7. W eissR A, W eiss M A. Controlled radi ofrequency endovenous occlusion usingauniqueradi ofrequency catheter under duplex guidance to eliminate saphenous varicose vein reflux: a 2-year follow-up. D ermatol Surg. 2002;28:38-42.

8. Rautio TT, Perala JM, W iik HT, Juvonen TS, H aukipuro $\mathrm{KA}$. Endovenous obliteration with radiofrequency-resistive heating for greater saphenous vein insufficiency: a feasibility study. J V asc Interv Radiol. 2002;13:569-75.

9. $\mathrm{M}$ in RJ, K hilnani $\mathrm{N}$, Zimmet SE. Endovenouslaser treatment of saphenous vein reflux: long-term results. J V asc Interv Radiol. 2003;14:991-6.

10. Chang $C, C$ hual. Endovenouslaser photocoagulation (EV LP) for varicose veins. Lasers Surg M ed. 2002;31:257-62.

Correspondência:

Jorge Enrique Soracco

Riobamba, 45120 "A"

Buenos Aires, Argentina

E-mail: jsoracco@fibertel.com.ar

O conteúdo do J Vasc Br etá disponível em português e em inglês

no ste do Jornal Vascular Brasileiro em

www.jvascbr.com.br 